\title{
Effects of diabetes-related stimuli on soluble epoxide hydrolase expression
}

\author{
Neeta Patwari ${ }^{1}$, Bomina Park ${ }^{1}$, Timothy Corson ${ }^{1}$ \\ ${ }^{1}$ Indiana University School of Medicine Department of Ophthalmology
}

Background and Hypothesis: Diabetic retinopathy (DR) is a frequent complication of diabetes and is a leading cause of blindness in the developed world. While the treatment of DR has progressed with the use of anti-VEGF therapies, the underlying mechanisms for DR are still unknown. Soluble epoxide hydrolase $(\mathrm{sEH})$ activity has been linked to retinal neovascularization, which can lead to blindness in proliferative DR. The use of sEH inhibitors also slowed the progression of experimental DR. In particular, sEH might be expressed in the retinal pigment epithelium (RPE), which is a critical player in metabolic processing and could be influenced by diabetes-related stimuli to promote neovascularization. We hypothesize that stimuli seen in diabetes can lead to sEH overexpression in human retinal pigmented epithelial cells (ARPE-19).

Experimental Design or Project Methods: To test our hypothesis, we examined $\mathrm{sEH}$ expression in ARPE-19 cells treated under hyperglycemic and hypoxic conditions similar to those of a diabetic retina. ARPE19 were treated with increasing doses of these stimuli and underwent RNA isolation, RT-PCR to study mRNA expression of $\mathrm{sEH}$, and immunoblotting to study protein expression of sEH.

Results After hyperglycemia treatment, there was a 1.5-fold increase in protein level of sEH from the control at $10 \mathrm{mM}$ glucose and a 1.2-fold increase at $20 \mathrm{mM}$ glucose. However, there was also a 0.5 -fold decrease in sEH protein level for the $50 \mathrm{mM}$ mannitol osmolarity control. These results require further replication. The hypoxia experiment is expected to show higher levels of sEH than the normoxia control and the mRNA expression analyses are underway.

Conclusion and Potential Impact: The results from this study suggest that hyperglycemic conditions can upregulate sEH expression in retinal pigment epithelial cells. Completion of this study will further our understanding of the underlying pathophysiology of DR. 